\title{
c-Recursion for multi-point superconformal blocks. NS sector
}

\author{
Vladimir Belavin ${ }^{a, b, c, 1}$ and Roman Geiko ${ }^{d, e}$ \\ ${ }^{a}$ I.E. Tamm Department of Theoretical Physics, P.N. Lebedev Physical Institute, \\ Leninsky ave. 53, 119991 Moscow, Russia \\ ${ }^{b}$ Department of Quantum Physics, Institute for Information Transmission Problems, \\ Bolshoy Karetny per. 19, 127994 Moscow, Russia \\ ${ }^{c}$ Department of Particle Physics and Astrophysics, Weizmann Institute of Science, \\ Rehovot 7610001, Israel \\ ${ }^{d}$ National Research University Higher School of Economics, \\ Usacheva str. 6, 119048 Moscow, Russia \\ ${ }^{e}$ Center for Advanced Studies, Skolkovo Institute of Science and Technology, \\ 143026 Moscow, Russia \\ E-mail: belavin@lpi.ru, romangeiko@mail.ru
}

Abstract: We develop a recursive approach to computing Neveu-Schwarz conformal blocks associated with n-punctured Riemann surfaces. This work generalizes the results of [1] obtained recently for the Virasoro algebra. The method is based on the analysis of the analytic properties of the superconformal blocks considered as functions of the central charge $c$. It consists of two main ingredients: the study of the singular behavior of the conformal blocks and the analysis of their asymptotic properties when $c$ tends to infinity. The proposed construction is applicable for computing multi-point blocks in different topologies. We consider some examples for genus zero and one with different numbers of punctures. As a by-product, we propose a new way to solve the recursion relations, which gives more efficient computational procedure and can be applied to SCFT case as well as to pure Virasoro blocks.

KeYwords: Conformal Field Theory, Conformal and W Symmetry

ARXIV EPRINT: 1806.09563

\footnotetext{
${ }^{1}$ Weston Visiting Professorship at Weizmann Institute.
} 


\section{Contents}

1 Introduction $\quad 1$

2 Preliminaries 3

2.1 NS sector fields 3

2.2 Matrix elements 5

3 Blocks on the sphere $\quad 7$

$\begin{array}{ll}3.1 & \text { Previous results: 4-point blocks }\end{array}$

3.2 Linear channel blocks 9

$\begin{array}{lll}3.3 & \text { Non-linear channel blocks } & 11\end{array}$

4 Blocks on the torus 12

4.1 Previous results: 1-point blocks 12

$\begin{array}{lll}4.2 & N \text {-point blocks in the necklace channel } & 13\end{array}$

5 Solutions of the recursion relations 14

$\begin{array}{llr}6 & \text { Discussion } & 16\end{array}$

$\begin{array}{lr}\text { A General } \operatorname{osp}(1 \mid 2) \text { matrix elements } & 16\end{array}$

$\begin{array}{lr}\text { B Some explicit coefficients } & 18\end{array}$

$\begin{array}{lr}\text { C Plumbing constructions } & 19\end{array}$

\section{Introduction}

It is well known that conformal blocks [2] are needed for computing correlation functions in any CFT model. Given that the space of local fields in the model consists of a set of irreducible representations of a symmetry algebra $\mathcal{A}_{\text {sym }}(\supset$ Virasoro algebra), conformal blocks are defined, using the concept of operator product expansion (OPE), as the holomorphic contribution to the correlation function coming from particular sets of representations in the intermediate OPE channels. Among conformal blocks the four-point blocks play most prominent role because they are needed for performing conformal bootstrap program (for the recent review, see [3]).

Most straightforwardly, conformal blocks can be expanded as a sum over irreducible representations (each consisting of a primary operator and its descendants) by inserting a complete set of states in each intermediate channel. For a given set of OPE channels 
this expansion corresponds to some special way of gluing three-punctured spheres (the socalled pant decomposition of the conformal blocks) and gives the expression of the blocks in terms of the matrix elements of chiral vertex operators between two states (primary or descendant). However this way has obvious technical restriction because it requires inversion of the matrix of scalar products of the descendant states, which in general is not diagonal and grows rapidly with the descendants' level. ${ }^{1}$

Another approach to computing conformal blocks is based on the study of their analytic properties. It has been initially invented in $[8,9]$ for computing four-point Virasoro blocks on the sphere by means of two types of recursions: the so-called $c$-recursion ( $c$ being the central charge parameter) and elliptic recursion (defined in terms of the intermediate conformal dimension $h$ ), exploiting analytic properties of the conformal blocks, considered as functions of $c$ and $h$ respectively. This allowed to verify the crossing relation for fourpoint correlation functions and thus to perform the bootstrap program in the Liouville theory [10]. Later these results have been extended to the case of $\mathcal{N}=1$ super-symmetric Liouville theory [11-13]. The generalization to the torus has been considered in [14, 15], however only one-point blocks have become available through the recursion constructions.

Meanwhile, the ability to compute conformal blocks is of interest from the $\mathrm{AdS}_{d+1} / \mathrm{CFT}_{d}$ perspective. One of the basic questions here is what AdS objects correspond to the boundary CFT conformal blocks. Significant progress in clarifying this question has been made in recent years [16-31] for $d=2$. It was shown that in the large $c$ regime the so-called heavy-light ${ }^{2}$ conformal blocks have clear geometric interpretation in terms of geodesic Witten diagrams. We notice that $c$-recursion fits naturally into $\mathrm{AdS}_{3} / \mathrm{CFT}_{2}$ context, because the large central charge limit, which is relevant for the semiclassical approximation in the dual gravity path integral, corresponds to the regular part of the conformal block in the $c$-recursion construction, as will be explained below. While the previous studies were based often on the direct matrix elements computation, more efficient methods of the recursion construction can serve as a useful tool for the further analysis of the correspondence. We notice that the sub-leading corrections in $c$ for higher multi-point correlation functions, especially on the torus, are basically not available from both the CFT and AdS sides, also the question of supersymmetric extenision of the $\mathrm{AdS}_{3} / \mathrm{CFT}_{2}$ correspondence remains almost unstudied (see, [35-37]).

Recently, it was shown [1] that Zamolodchikov's analysis of the Virasoro CFT can be also applied for computing higher multi-point correlators on the sphere and torus and, in principle, on higher genius Riemann surfaces. In this paper we develop analogous techniques for multi-point blocks in $\mathcal{N}=1$ super-Virasoro (SVir) field theory. We focus on the Neveu-Schwarz (NS) sector of the theory (for precise definition see section 2).

The analytic properties with respect to $c$ give rise to the splitting of SVir blocks into two parts: the regular part, corresponding to the limit $c \rightarrow \infty$ (the so-called light asymptotic),

\footnotetext{
${ }^{1}$ Using AGT correspondence [4] the problem of non-diagonality can be solved for (super-)Virasoro algebra by extending [5] the symmetry algebra and introducing the so-called integrable basis [6, 7], however the rapid growth problem still remains.

${ }^{2}$ The term referring to a certain behavior [32] of the conformal dimensions in the limit $c \rightarrow \infty$. For other possible regimes in this context, see, e.g., [33, 34].
} 
and the singular part, coming from (in general) simple poles, located at degenerate values of the intermediate conformal dimensions, $d_{r, s}(c)$. On the sphere the regular part is governed by $\operatorname{osp}(1 \mid 2)$ subalgebra of SVir, so that the light SVir blocks on the sphere are reduced to the blocks of $\operatorname{ssp}(1 \mid 2)$ (the so-called global blocks). The light blocks on the torus are a bit more involved objects: they can be factorized on the global osp (1|2) blocks on the torus and NS vacuum characters.

Another approach to compute Virasoro blocks, different from the mentioned above, has been proposed in [38]. This approach is based on decomposing contributions of conformal families into sums over modules growing from quasiprimary states. This allows to express conformal blocks as a sum of light blocks with shifted intermediate dimensions. This representation gives in fact the solution of $c$-recursion relation for the Virasoro 4-point block and provides in principle more efficient way to compute general blocks.

In the present work we consider the $N$-point SVir conformal blocks on the sphere in the linear channel (including 4-point case, for the terminology see 3.2) and provide $c$-recursion formulas for them. Then, we consider non-linear OPE channel and obtain $c$-recursion formulas for the correspondind SVir block. Further, we provide the c-recursion for the n-point SVir block in a necklace channel on the torus (including 1-point case). Using the approach of [38] we obtain the solutions of $c$-recursion relations in the considered cases. In section 2 we recall some facts about NS sector of $\mathcal{N}=1$ super-Virasoro algebra and its modules.

In sections 3 and 4 we analyze the recursion relations for superconformal blocks on the sphere and torus respectively. In section 5 we discuss an improvement, that allows to resolve $c$-recursion formulas. Some technical details are collected in the appendices.

\section{Preliminaries}

In this section we briefly remind some facts about NS sector of $\mathcal{N}=1 \mathrm{CFT}$, which are relevant for our purposes. For more systematic exposition and the general landscape, see, e.g., $[13,39]$.

\subsection{NS sector fields}

The fields of $\mathcal{N}=1$ CFT belong to highest weight representations (modules) of the superconformal algebra ${ }^{3}$

$$
\begin{aligned}
{\left[L_{n}, L_{m}\right] } & =(n-m) L_{m+n}+\frac{c}{8} n\left(n^{2}-1\right) \delta_{m+n, 0}, \\
{\left[L_{n}, G_{k}\right] } & =\left(\frac{n}{2}-k\right) G_{n+k} \\
\left\{G_{k}, G_{l}\right\} & =2 L_{k+l}+\frac{c}{2}\left(k^{2}-\frac{1}{4}\right) \delta_{k+l, 0},
\end{aligned}
$$

where in the NS sector

$$
n, m \in Z \quad \text { and } \quad k, l \in \mathbb{Z}+\frac{1}{2}
$$

and $c$ is the central charge.

\footnotetext{
${ }^{3}$ Because the object of our study is conformal blocks, from now on we neglect the second (antiholomorphic) copy of the symmetry algebra and suppress the dependence on $\bar{z}$.
} 
The highest weight vector or the primary state $|d\rangle$ is defined as

$$
\begin{aligned}
L_{0}|d\rangle & =d|d\rangle, \\
L_{n}|d\rangle & =0, n>0, \\
G_{k}|d\rangle & =0, k>0,
\end{aligned}
$$

where $d$ is the conformal dimension parameter. In the Liouville-like $(\lambda, b)$-parametrization, the conformal weight of the primary state is given by

$$
d(\lambda)=\frac{\left(b+b^{-1}\right)^{2}}{8}-\frac{\lambda^{2}}{8},
$$

and

$$
c(b)=1+2\left(b+b^{-1}\right)^{2} .
$$

The module $\mathcal{H}_{d}$ is spanned by the states

$$
\mathcal{L}_{\vec{k}}|d\rangle=G_{-l_{1}} \ldots G_{-l_{p}} L_{-n_{1}} \ldots L_{-n_{m}}|d\rangle
$$

where $0<l_{1}<\ldots<l_{p}$ and $0<n_{1} \leq \ldots \leq n_{m}$. The grading with respect to $L_{0}$ :

$$
L_{0} \mathcal{L}_{\vec{k}}|d\rangle=k \mathcal{L}_{\vec{k}}|d\rangle, \quad \text { with } k=\sum_{i=1}^{p} l_{i}+\sum_{j=1}^{m} n_{j},
$$

defines the level $k \in \mathbb{N}_{0}+\frac{1}{2}$ of the descendant state $\mathcal{L}_{\vec{k}}|d\rangle$. The states $|d\rangle$ and $G_{-\frac{1}{2}}|d\rangle$ correspond, respectively, to the (lower and upper) components of the primary supermultiplet $V_{d}(z)$ and $\widetilde{V}_{d}(z)$. The conjugation is defined by

$$
\langle d| \mathcal{L}_{\vec{k}}^{\dagger}=\langle d| L_{n_{m}} \ldots L_{n_{1}} G_{l_{p}} \ldots G_{l_{1}} .
$$

We denote by $B_{\vec{k}_{1}, \vec{k}_{2}}(d, c),{ }^{4}$ the matrix of scalar products

$$
B_{\vec{k}_{1}, \vec{k}_{2}}=\left\langle d\left|\mathcal{L}_{\vec{k}_{1}}^{\dagger} \mathcal{L}_{\vec{k}_{2}}\right| d\right\rangle
$$

and fix the normalization condition $\langle d \mid d\rangle=1$. The matrix of scalar products has block diagonal structure $B_{\vec{k}_{1}, \vec{k}_{2}} \sim \delta_{k_{1}, k_{2}}$ with the size $P_{\mathrm{NS}}(k)$ of $k$-th level block defined by the NS character

$$
\sum_{k \in \frac{1}{2} \mathbb{N}_{0}} P_{\mathrm{NS}}(k) q^{k}=\prod_{p=1}^{\infty} \frac{1+q^{p-\frac{1}{2}}}{1-q^{p}} .
$$

For the special values $d_{r, s}:=d\left(\lambda_{r, s}\right)$, where $\lambda_{r, s}=r b+s b^{-1}, r, s \in \mathbb{Z}_{+}$and $r+s$ is even, the NS module $\mathcal{H}_{d}$ becomes degenerated - the $\frac{r s}{2}$-th level contains a singular vector $\chi_{r, s} \cdot{ }^{5}$ Restricted on the $n$-th level, the Kac determinant is given by

$$
\operatorname{det} B^{(n)}=\prod_{1 \leq r s \leq 2 n}\left(d-d_{r, s}\right)^{P_{\mathrm{NS}}\left(n-\frac{r s}{2}\right)} .
$$

Hence, for $n \geq \frac{r s}{2}$, in the module $\mathcal{H}_{d}$ with $d=d_{r, s}(c)$, the Kac $\operatorname{determinant} \operatorname{det} B^{(n)}=0$.

\footnotetext{
${ }^{4}$ We shall omit one or both arguments of $B_{\vec{k}_{1}, \vec{k}_{2}}(d, c)$, when it does not lead to confusion.

${ }^{5}$ In what follows we use the normalization of the singular vectors with coefficient 1 in front of $G_{-\frac{1}{2}}^{r s} \nu_{r, s}$.
} 


\section{$2.2 \quad$ Matrix elements}

Below we denote by $\nu_{i}$ and $V_{i}(z) \equiv V_{d_{i}}(z)$ the primary state $\left|d_{i}\right\rangle$ and the corresponding vertex operator. The matrix elements of a general vertex operator can be regarded as three-linear forms:

$$
\rho(., ., .): \mathcal{H}^{3} \rightarrow \mathbb{C}, \quad \rho\left(\mathcal{L}_{\vec{k}_{1}} \nu_{1}, \mathcal{L}_{\vec{k}_{2}} \nu_{2}, \mathcal{L}_{\vec{k}_{3}} \nu_{3}\right)=\left.\left\langle d_{1}\left|\mathcal{L}_{\vec{k}_{1}}^{\dagger}\left(\mathcal{L}_{\vec{k}_{2}} V_{2}(z)\right) \mathcal{L}_{\vec{k}_{3}}\right| d_{3}\right\rangle\right|_{z \rightarrow 1} .
$$

Here we assume the following normalization condition:

$$
\left\langle d_{1}\left|V_{2}(z)\right| d_{3}\right\rangle=z^{d_{1}-d_{2}-d_{3}}, \quad\left\langle d_{1}\left|\widetilde{V}_{2}(z)\right| d_{3}\right\rangle=z^{d_{1}-d_{2}-d_{3}-\frac{1}{2}} .
$$

The commutation relations between super-Virasoro generators and vertex operators:

$$
\begin{aligned}
{\left[L_{n}, V_{d}(z)\right] } & =z^{n}\left(z \partial_{z}+(n+1) d\right) V_{d}(z), \\
{\left[L_{n}, \tilde{V}_{d}(z)\right] } & =z^{n}\left(z \partial_{z}+(n+1)\left(d+\frac{1}{2}\right)\right) V_{d}(z), \\
{\left[G_{k}, V_{d}(z)\right] } & =z^{k+\frac{1}{2}} \tilde{V}_{d}(z), \\
\left\{G_{k}, \tilde{V}_{d}(z)\right\} & =z^{k-\frac{1}{2}}\left(z \partial_{z}+d(2 k+1)\right) V_{d}(z),
\end{aligned}
$$

allows one to express the matrix elements involving arbitrary descendants in terms of the two independent matrix elements $\rho^{\alpha_{2}}\left(\nu_{1}, \nu_{2}, \nu_{3}\right)$, where $\alpha_{2} \in\{0,1\}$ stand for the upper and lower component of $\nu_{2}$ respectively,

$$
\rho^{0}\left(\nu_{1}, \nu_{2}, \nu_{3}\right):=\rho\left(\nu_{1}, \nu_{2}, \nu_{3}\right) \quad \text { and } \quad \rho^{1}\left(\nu_{1}, \nu_{2}, \nu_{3}\right):=\rho\left(\nu_{1}, G_{-\frac{1}{2}} \nu_{2}, \nu_{3}\right) .
$$

The matrix elements of singular vectors $\rho\left(\chi_{r, s}(c), .,.\right)$ must vanish if superconformal fusion rules $\nu_{r, s} \times \nu_{2} \rightarrow \nu_{3}$ are satisfied and therefore can be expressed in terms of the so-called fusion polynomials $[11,12]$

$$
\begin{array}{r}
P_{r, s}^{0}\left(d_{i}, d_{j} ; c\right)=\prod_{\substack{p=1-r \\
\text { step 2 } 2}}^{r-1} \prod_{\substack{q=1-s \\
\text { step 2 }}}^{s-1}\left(\frac{\lambda_{i}-\lambda_{j}+p b+q b^{-1}}{2 \sqrt{2}}\right)\left(\frac{\lambda_{i}+\lambda_{j}+p b+q b^{-1}}{2 \sqrt{2}}\right), \\
p+q-(r+s) \in 4 \mathbb{Z}+2, \\
P_{r, s}^{1}\left(d_{i}, d_{j} ; c\right)=\prod_{\substack{p=1-r \\
\text { step 2 }}}^{r-1} \prod_{\substack{q=1-s \\
\text { step 2 }}}^{s-1}\left(\frac{\lambda_{i}-\lambda_{j}+p b+q b^{-1}}{2 \sqrt{2}}\right)\left(\frac{\lambda_{i}+\lambda_{j}+p b+q b^{-1}}{2 \sqrt{2}}\right), \\
p+q-(r+s) \in 4 \mathbb{Z},
\end{array}
$$

where subscript $c$ indicates the dependence on the central charge parameter, which comes through the parameter $b$ according to (2.4). We denote ${ }^{6}$

$$
\rho^{\alpha}\left(\chi_{r, s}(c), \nu_{i}, \nu_{j}\right)=\sigma_{r, s}^{\alpha}\left(d_{i}, d_{j} ; c\right),
$$

\footnotetext{
${ }^{6}$ Other types of matrix elements with singular vectors, like $\rho\left(\chi_{r, s}(c), \nu_{i}, G_{-\frac{1}{2}} \nu_{j}\right)$, etc., are not linear independent and will not be relevant.
} 
then

$$
\sigma_{r, s}^{\alpha}\left(d_{i}, d_{j} ; c\right)= \begin{cases}P_{r, s}^{\alpha}\left(d_{i}, d_{j} ; c\right), & r s \in 2 \mathbb{N}, \\ P_{r, s}^{t(\alpha)}\left(d_{i}, d_{j} ; c\right), & r s \in 2 \mathbb{N}+1,\end{cases}
$$

where $t(0)=1$ and $t(1)=0$. Taking into account reflection symmetry:

$$
\begin{aligned}
\rho\left(\mathcal{L}_{\vec{k}} \nu_{1}, \nu_{2}, \nu_{3}\right) & =\rho\left(\nu_{3}, \nu_{2}, \mathcal{L}_{\vec{k}} \nu_{1}\right), & & k \in 2 \mathbb{N}_{0}, \\
\rho\left(\mathcal{L}_{\vec{k}} \nu_{1}, G_{-\frac{1}{2}} \nu_{2}, \nu_{3}\right) & =\rho\left(\nu_{3}, G_{-\frac{1}{2}} \nu_{2}, \mathcal{L}_{\vec{k}} \nu_{1}\right), & & k \in 2 \mathbb{N}_{0}, \\
\rho\left(\mathcal{L}_{\vec{k}} \nu_{1}, \nu_{2}, \nu_{3}\right) & =\rho\left(\nu_{3}, \nu_{2}, \mathcal{L}_{\vec{k}} \nu_{1}\right), & & k \in 2 \mathbb{N}_{0}+1, \\
\rho\left(\mathcal{L}_{\vec{k}} \nu_{1}, G_{-\frac{1}{2}} \nu_{2}, \nu_{3}\right) & =-\rho\left(\nu_{3}, G_{-\frac{1}{2}} \nu_{2}, \mathcal{L}_{\vec{k}} \nu_{1}\right), & & k \in 2 \mathbb{N}_{0}+1,
\end{aligned}
$$

which follows from the conjugation relation (2.7) and the minus sign in the last relation is due to anticommutativity of the odd operators, one gets

$$
\rho^{\alpha}\left(\nu_{i}, \nu_{j}, \chi_{r, s}(c)\right)=S_{r s}^{\alpha} \sigma_{r, s}^{\alpha}\left(d_{j}, d_{i} ; c\right),
$$

where

$$
S_{n}^{0}=1, \quad S_{n}^{1}=(-1)^{n} .
$$

Now we find the matrix element with two identical singular vectors $\mu_{r, s}^{\alpha}(d ; c):=$ $\rho^{\alpha}\left(\chi_{r, s}, \nu_{d}, \chi_{r, s}\right)$, which reads

$$
\mu_{r, s}^{\alpha}(d, c)= \begin{cases}P_{r, s}^{\alpha}\left(d, d_{r, s}+\frac{r s}{2} ; c\right) P_{r, s}^{\alpha}\left(d, d_{r, s} ; c\right), & r s \in 2 \mathbb{N}, \\ S_{r s}^{\alpha} P_{r, s}^{t(\alpha)}\left(d, d_{r, s}+\frac{r s}{2} ; c\right) P_{r, s}^{\alpha}\left(d, d_{r, s} ; c\right), & r s \in 2 \mathbb{N}+1 .\end{cases}
$$

Here and below we use the following factorization property

$$
\rho^{\alpha}\left(\mathcal{L}_{\vec{k}} \chi_{r, s}, \nu_{i}, \nu_{j}\right)=\rho^{\alpha}\left(\mathcal{L}_{\vec{k}} \nu_{d_{r, s}+\frac{r s}{2}}, \nu_{i}, \nu_{j}\right) \rho^{\alpha}\left(\chi_{r, s}, \nu_{i}, \nu_{j}\right),
$$

and, in particular, we use

$$
\rho^{\alpha}\left(\mathcal{L}_{\vec{k}} \chi_{r, s}, \nu_{d}, \mathcal{L}_{\vec{k}} \chi_{r, s}\right)=\mu_{r, s}^{\alpha}(d ; c) \rho^{\alpha}\left(\mathcal{L}_{\vec{k}} \nu_{d_{r, s}+\frac{r s}{2}}, \nu_{d}, \mathcal{L}_{\vec{k}} \nu_{d_{r, s}+\frac{r s}{2}}\right)
$$

$\boldsymbol{h}$-parametrization. Alternatively, we can consider the matrix elements of singular vectors $\rho\left(\chi_{r, s}(c), .,.\right)$ as functions of the conformal dimension $h$ of the primary state $\nu_{h}$ possessing the singular descendant. To this end we use

$$
c_{r, s}(h)=1+2\left(b_{r, s}+b_{r, s}^{-1}\right)^{2},
$$

and

$$
h=\frac{\left(b_{r, s}+b_{r, s}^{-1}\right)^{2}}{8}-\frac{\left(r b_{r, s}+s b_{r, s}^{-1}\right)^{2}}{8} .
$$

We define $P_{r, s}^{\alpha}\left(d_{i}, d_{j} ; h\right):=P_{r, s}^{\alpha}\left(d_{i}, d_{j} ; c_{r, s}(h)\right)$ by means of (2.18) and (2.19) with $b$ parameter replaced by $b_{r, s}(h)$. The matrix elements in the $h$-parametrization are given by

$$
\sigma_{r, s}^{\alpha}\left(d_{i}, d_{j} ; h\right):=\sigma_{r, s}^{\alpha}\left(d_{i}, d_{j} ; c_{r, s}(h)\right), \quad \mu_{r, s}^{\alpha}(d ; h):=\mu_{r, s}^{\alpha}\left(d ; c_{r, s}(h)\right) .
$$




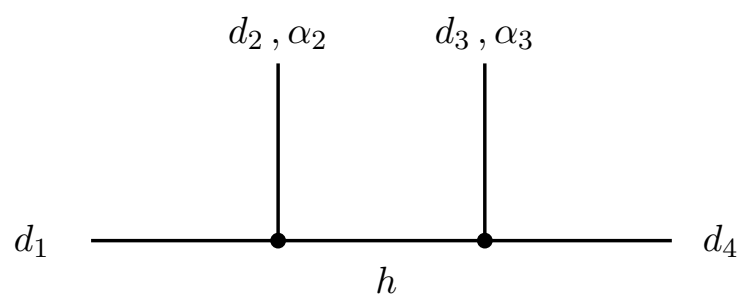

Figure 1. Dual diagram for basic 4-point superconformal blocks on the sphere.

\section{Blocks on the sphere}

In this and the subsequent sections we use the following convention: the matrix elements contributing to the conformal block correspond to the vertices of the associated dual diagram, the vertex operators correspond to vertical lines, while asymptotic states correspond to horizontal lines. We denote by $\alpha_{i}=0,1$ - lower and upper components of $\nu_{i}$ respectively, where $i$ numerates external lines. To be more compact, we suppress dependence on $c$ and conformal dimensions, as well as coordinate dependence, of the conformal blocks. Also we suppress obvious conformal prefactors. We will use unique notation $F, G$ for blocks considered in each subsection, referring to the corresponding diagrammatic representation.

\subsection{Previous results: 4-point blocks}

First we recapitulate the $c$-recursive representation for 4-point superconformal blocks, which has been developed in $[11,12]$. Using super-projective invariance, we can chose among 16 blocks of lower and upper components of primary supermultiplets (in the given OPE channel) four linear independent blocks according to the diagram depicted in figure 1.

These blocks have the following series expansion

$$
F=\sum_{n \in \frac{1}{2} \mathbb{N}_{0}} F_{n} z^{n}
$$

where $z$ is anharmonic ratio of the holomorhic coordinates (in our convention $\nu_{1}, \nu_{2}, \nu_{4}$ stand at $\infty, 1,0$ respectively) and the coefficients are given by

$$
F_{n}=\sum_{\substack{k=m \\ m=n}} B^{\vec{k}, \vec{m}} \rho^{\alpha_{2}}\left(\nu_{1}, \nu_{2}, \mathcal{L}_{\vec{k}} \nu_{h}\right) \rho^{\alpha_{3}}\left(\mathcal{L}_{\vec{m}} \nu_{h}, \nu_{3}, \nu_{4}\right) .
$$

Here the sum goes over (half-)integer partitions and $B^{\vec{k}, \vec{m}}$ is the inverse Gram matrix.

The determinant of the Gram matrix has zeros at $h=d_{r, s}$. We consider the residues of the conformal block at the corresponding poles. The coefficients $F_{n}$ for $n \geq \frac{r s}{2}$ have poles at $h=d_{r, s}$. In the limit $h \rightarrow d_{r, s}$ the matrix elements entering (3.2) are non-singular and factorize according to (2.26). The singular vector $\chi_{r, s}$ is obtained by applying $\mathcal{L}_{r, s}$ to the degenerated state $\nu_{r, s}$. We denote by $\chi_{r, s}^{h}$ the state, which is obtained by applying $\mathcal{L}_{r, s}$ to $\nu_{h}$ and which is the only one giving contribution to the residue of $B^{\vec{k}, \vec{m}}$. Using factorization 
property (2.26), we get

$$
\begin{aligned}
& \lim _{h \rightarrow d_{r, s}}\left(h-d_{r, s}\right)\left\langle\chi_{r, s}^{h}\left|\mathcal{L}_{\vec{k}}^{\dagger} \mathcal{L}_{\vec{m}}\right| \chi_{r, s}^{h}\right\rangle^{-1} \\
& =\left\langle\nu_{d_{r, s}+\frac{r s}{2}}\left|\mathcal{L}_{\vec{k}}^{\dagger} \mathcal{L}_{\vec{m}}\right| \nu_{d_{r, s}+\frac{r s}{2}}\right\rangle^{-1} \lim _{h \rightarrow d_{r, s}}\left(h-d_{r, s}\right)\left\langle\chi_{r, s}^{h} \mid \chi_{r, s}^{h}\right\rangle^{-1}=B^{\vec{k}, \vec{m}}\left(d_{r, s}+\frac{r s}{2}\right) A_{r, s}(c),
\end{aligned}
$$

where the coefficient

$$
A_{r, s}(c)=\frac{1}{2} \prod_{p=1-r}^{r} \prod_{q=1-s}^{s}\left(\frac{1}{\sqrt{2}}\left(p b+q b^{-1}\right)\right)^{-1}, \quad p+q \in 2 \mathbb{Z}, \quad(p, q) \neq(0,0),(r, s) .
$$

Assembling all the ingredients we get the following relation

$$
\lim _{h \rightarrow d_{r, s}}\left(h-d_{r, s}\right) F=z^{\frac{r s}{2}} A_{r, s}(c) S_{r s}^{\alpha_{2}} \sigma_{r, s}^{\alpha_{2}}\left(d_{2}, d_{1} ; c\right) \sigma_{r, s}^{\alpha_{3}}\left(d_{3}, d_{4} ; c\right) F\left(h \rightarrow d_{r, s}+\frac{r s}{2}, c\right) .
$$

Considering the residues of the conformal block at $c=c_{r, s}(h)$, we have

$$
\begin{aligned}
\lim _{c \rightarrow c_{r, s}}\left(c-c_{r, s}(h)\right) F= & z^{\frac{r s}{2}} J_{r, s}(h) A_{r, s}(h) S_{r s}^{\alpha_{2}} \sigma_{r, s}^{\alpha_{2}}\left(d_{2}, d_{1} ; h\right) \sigma_{r, s}^{\alpha_{3}}\left(d_{3}, d_{4} ; h\right) \\
& \times F\left(h \rightarrow h+\frac{r s}{2}, c \rightarrow c_{r, s}(h)\right),
\end{aligned}
$$

where Jacobian $J_{r, s}:=-\frac{\partial c_{r, s}}{\partial h}$ and

$$
A_{r, s}(h)=\frac{1}{2} \prod_{p=1-r}^{r} \prod_{q=1-s}^{s}\left(\frac{1}{\sqrt{2}}\left(p b_{r, s}+q b_{r, s}^{-1}\right)\right)^{-1}, \quad p+q \in 2 \mathbb{Z}, \quad(p, q) \neq(0,0),(r, s) .
$$

In order to obtain the regular part or the light block we take the large $c$ limit of the conformal block keeping all the dimensions fixed. The contribution of the global subalgebra is dominating in this limit and the light NS super-Virasoro block $G$ is nothing but the conformal block associated to the $\operatorname{osp}(1 \mid 2)$ subalgebra

$$
\begin{aligned}
G=\sum_{n \in \frac{1}{2} \mathbb{N}_{0}} G_{n} z^{n} & =\sum_{n \in \mathbb{N}_{0}} \sum_{\beta \in\{0,1\}} \frac{\rho^{0, \alpha_{2}, \beta}\left(\nu_{1}, \nu_{2}, L_{-1}^{n} \nu_{h}\right) \rho^{\beta, \alpha_{3}, 0}\left(L_{-1}^{n} \nu_{h}, \nu_{3}, \nu_{4}\right)}{\rho^{\beta, 0, \beta}\left(L_{-1}^{n} \nu_{h}, \mathbb{1}, L_{-1}^{n} \nu_{h}\right)} z^{n+\frac{\beta}{2}} \\
& =\sum_{n \in \mathbb{N}_{0}} \sum_{\beta \in\{0,1\}} \frac{\left(h+\frac{\beta}{2}+d_{2}-d_{1}\right)_{n}\left(h+\frac{\beta}{2}+d_{3}-d_{4}\right)_{n}}{n !(2 h)_{n+\beta}} z^{n+\frac{\beta}{2}},
\end{aligned}
$$

where $\mathbb{1}$ stands for the unity operator with $d=0$. The definition of $\operatorname{ssp}(1 \mid 2)$ algebra and the explicit global matrix elements can be found ${ }^{7}$ in appendix A. For the four-point block's coefficients we get the following recursion

$$
F_{n}=G_{n}+\sum_{\substack{r \geq 2, s \geq 1 \\ r+s \in 2 \mathbb{N}}}^{r s \leq 2 n} \frac{R_{r, s}(h)}{c-c_{r, s}(h)} F_{n-\frac{r s}{2}}\left(h \rightarrow h+\frac{r s}{2}, c \rightarrow c_{r, s}(h)\right),
$$

where

$$
R_{r, s}(h)=J_{r, s}(h) A_{r, s}(h) S_{r s}^{\alpha_{3}} \sigma_{r, s}^{\alpha_{2}}\left(d_{1}, d_{2} ; h\right) \sigma_{r, s}^{\alpha_{3}}\left(d_{4}, d_{3} ; h\right) .
$$

\footnotetext{
${ }^{7}$ For another approach to the analysis of the light asymptotic, based on AGT correspondence, see [40].
} 


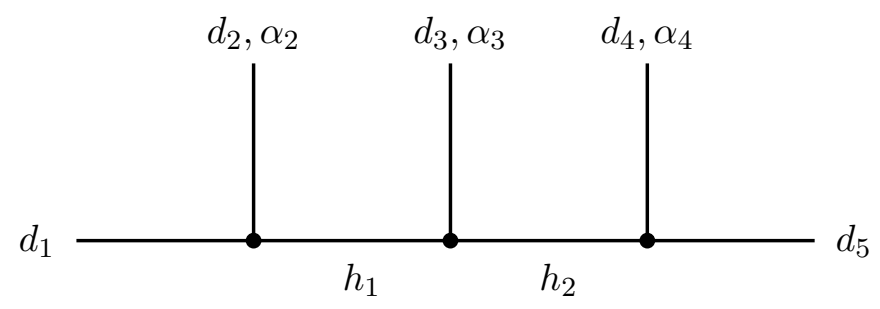

Figure 2. 5-point superconformal block in the linear channel.

\subsection{Linear channel blocks}

5-point block in the linear channel. In what follows we call an OPE channel linear if it corresponds to the insertion of the complete set of states between each successive pair of external operators as depicted on figure 3 , the channels of other types we call non-linear (see e.g. figure 4). Here we derive the recursion formulas for the 5-point conformal block in the linear OPE channel represented in figure 2.

In this case using super-projective invariance we can fix $\nu_{1}$ and $\nu_{5}$ to be lower primary components and to put operators $\nu_{1}, \nu_{2}, \nu_{5}$ at positions $\infty, 1,0$ respectively. It is possible to construct a local parametrization $q_{i}$ of the moduli space of Riemann sphere with $N$ punctures located at $z_{i}$, so that conformal blocks have Taylor series expansion in $q_{i}$ (see appendix $\mathrm{C}$ for the construction in this particular case). The conformal block reads

$$
\begin{aligned}
F=\sum_{n_{1}, n_{2} \in \frac{1}{2} N_{0}} F_{n_{1}, n_{2}} q_{1}^{n_{1}} q_{2}^{n_{2}}= & \sum_{\vec{k}_{1,2}, \vec{p}_{1,2}} q_{1}^{k_{1}} q_{2}^{k_{2}} B^{\vec{k}_{1}, \vec{p}_{1}}\left(h_{1}\right) B^{\vec{k}_{2}, \vec{p}_{2}}\left(h_{2}\right) \times \\
& \times \rho^{\alpha_{2}}\left(\nu_{1}, \nu_{2}, \mathcal{L}_{\vec{k}_{1}} \nu_{h_{1}}\right) \rho^{\alpha_{3}}\left(\mathcal{L}_{\vec{p}_{1}} \nu_{h_{2}}, \nu_{3}, \mathcal{L}_{\vec{k}_{2}} \nu_{h_{2}}\right) \rho^{\alpha_{4}}\left(\mathcal{L}_{\vec{p}_{2}} \nu_{h_{2}}, \nu_{4}, \nu_{5}\right),
\end{aligned}
$$

where, as explained in appendix C, $q_{1}=z_{3}, q_{2}=\frac{z_{4}}{z_{3}}$. Analyzing singular behavior of the conformal block in the both intermediate channels, we get

$$
\begin{aligned}
F_{n_{1}, n_{2}}= & G_{n_{1}, n_{2}}+\sum_{\substack{r \geq 2, s \geq 1 \\
r+s \in 2 \mathbb{N}}}^{r s \leq 2 n_{1}} \frac{R_{r, s}^{1}\left(h_{1}\right)}{c-c_{r, s}\left(h_{1}\right)} F_{n_{1}-\frac{r s}{2}, n_{2}}\left(h_{1} \rightarrow h_{r, s}+\frac{r s}{2}, c \rightarrow c_{r, s}\right) \\
& +\sum_{\substack{r \geq 2, s \geq 1, r+s \in 2 \mathbb{N}}}^{r s \leq 2 n_{2}} \frac{R_{r, s}^{2}\left(h_{2}\right)}{c-c_{r, s}\left(h_{2}\right)} F_{n_{1}, n_{2}-\frac{r s}{2}}\left(h_{2} \rightarrow h_{r, s}+\frac{r s}{2}, c \rightarrow c_{r, s}\right),
\end{aligned}
$$

where coefficients $R_{r, s}^{i}$ are the following

$$
\begin{aligned}
& R_{r, s}^{1}\left(h_{1}\right)=J_{r, s}\left(h_{1}\right) A_{r, s}\left(h_{1}\right) S_{r s}^{\alpha_{2}} \sigma_{r, s}^{\alpha_{2}}\left(d_{2}, d_{1} ; h_{1}\right) \sigma_{r, s}^{\alpha_{3}}\left(d_{3}, h_{2} ; h_{1}\right), \\
& R_{r, s}^{2}\left(h_{2}\right)=J_{r, s}\left(h_{2}\right) A_{r, s}\left(h_{2}\right) S_{r s}^{\alpha_{3}} \sigma_{r, s}^{\alpha_{3}}\left(d_{3}, h_{1} ; h_{2}\right) \sigma_{r, s}^{\alpha_{4}}\left(d_{4}, d_{5} ; h_{2}\right) .
\end{aligned}
$$




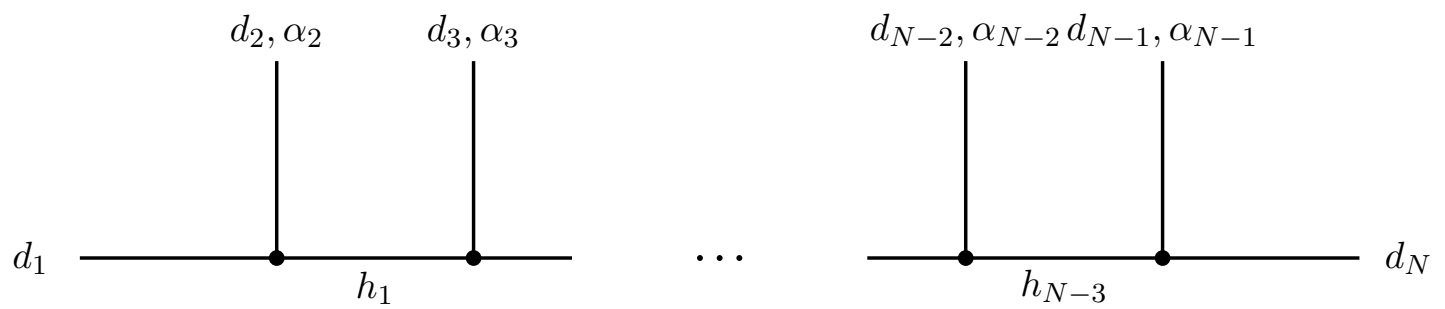

Figure 3. $N$-point conformal block in the linear channel.

Using the results of appendix A, we find explicit coefficients of the 5-point light block

$$
\begin{aligned}
G= & \sum_{k, m \in \frac{1}{2} \mathbb{N}_{0}} G_{k, m} q_{1}^{k} q_{2}^{m}=\sum_{k, m \in \mathbb{N}_{0}} \sum_{\beta_{1,2} \in\{0,1\}} q_{1}^{k+\frac{\beta_{1}}{2} q_{2}^{m+\frac{\beta_{2}}{2}} \times} \\
& \times \frac{\rho^{0, \alpha_{2}, \beta_{1}}\left(\nu_{1}, \nu_{2}, L_{-1}^{k} \nu_{h_{1}}\right) \rho^{\beta_{1}, \alpha_{3}, \beta_{2}}\left(L_{-1}^{k} \nu_{h_{1}}, \nu_{3}, L_{-1}^{m} \nu_{h_{2}}\right) \rho^{\beta_{2}, \alpha_{4}, 0}\left(L_{-1}^{m} \nu_{h_{2}}, \nu_{4}, \nu_{5}\right)}{\rho^{\beta_{1}, 0, \beta_{1}}\left(L_{-1}^{k} \nu_{h_{1}}, \mathbb{1}, L_{-1}^{k} \nu_{h_{1}}\right) \rho^{\beta_{2}, 0, \beta_{2}}\left(L_{-1}^{m} \nu_{h_{2}}, \mathbb{1}, L_{-1}^{m} \nu_{h_{2}}\right)} \\
= & \sum_{k, m \in \mathbb{N}_{0}} \sum_{\beta_{1,2} \in\{0,1\}} q_{1}^{k+\frac{\beta_{1}}{2}} q_{2}^{m+\frac{\beta_{2}}{2}} \frac{\left(h_{1}+\frac{\beta_{1}}{2}+d_{2}-d_{1}\right)_{k}\left(h_{2}+\frac{\beta_{2}}{2}+d_{4}-d_{5}\right)_{m}}{k ! m !\left(2 h_{1}\right)_{k+\beta_{1}}\left(2 h_{2}\right)_{m+\beta_{2}}} \tau_{k, m}^{\beta_{1}, \alpha_{3}, \beta_{2}}\left(h_{1}, d_{3}, h_{2}\right) .
\end{aligned}
$$

$N$-point block in the linear channel. Below we generalize the previous consideration to $N$-point block in the linear channel.

From figure 3 we read off the conformal block

$$
\begin{aligned}
F=\sum_{n_{i} \in \frac{1}{2} N_{0}} F_{n_{1}, \ldots, n_{N-3}} q_{1}^{n_{1}} \ldots q_{N-3}^{n_{N-3}}= & \sum_{\vec{k}_{i}, \vec{p}_{i}} q_{1}^{k_{1}} \ldots q_{N-3}^{k_{N-3}} B^{\vec{k}_{1}, \vec{p}_{1}}\left(h_{1}\right) \ldots B^{\vec{k}_{N-3}, \vec{p}_{N-3}}\left(h_{N-3}\right) \times \\
& \times \rho^{\alpha_{2}}\left(\nu_{1}, \nu_{2}, \mathcal{L}_{\vec{k}_{1}} \nu_{h_{1}}\right) \ldots \rho^{\alpha_{N-1}}\left(\mathcal{L}_{\vec{p}_{N-3}} \nu_{h_{N-3}}, \nu_{N-1}, \nu_{N}\right),
\end{aligned}
$$

where moduli parameters are related to the positions of the vertex operators through

$$
q_{1}=z_{3}, \quad q_{i}=\frac{z_{i+2}}{z_{i+1}}, \quad 2 \leq i \leq N-3
$$

and we fix $z_{1}=\infty, z_{2}=1, z_{N}=0$ (see appendix C, figure 7).

One gets the following recursion representation

$$
\begin{aligned}
F_{n_{1}, \ldots, n_{N-3}}= & G_{n_{1}, \ldots, n_{N-3}} \\
& +\sum_{i=1}^{N-3} \sum_{\substack{r \geq 2, s \geq 1 \\
r_{i}+s_{i} \in 2 \mathrm{~N}}}^{r_{i} s_{i} \leq 2 n_{i}} \frac{R_{r_{i}, s_{i}}^{i}\left(h_{i}\right)}{c-c_{r_{i}, s_{i}}\left(h_{i}\right)} F_{n_{1}, \ldots, n_{i}-\frac{r_{i} s_{i}}{2}, \ldots, n_{N-3}}\left(h_{i} \rightarrow h_{r_{i}, s_{i}}+\frac{r_{i} s_{i}}{2}, c \rightarrow c_{r_{i}, s_{i}}\left(h_{i}\right)\right),
\end{aligned}
$$




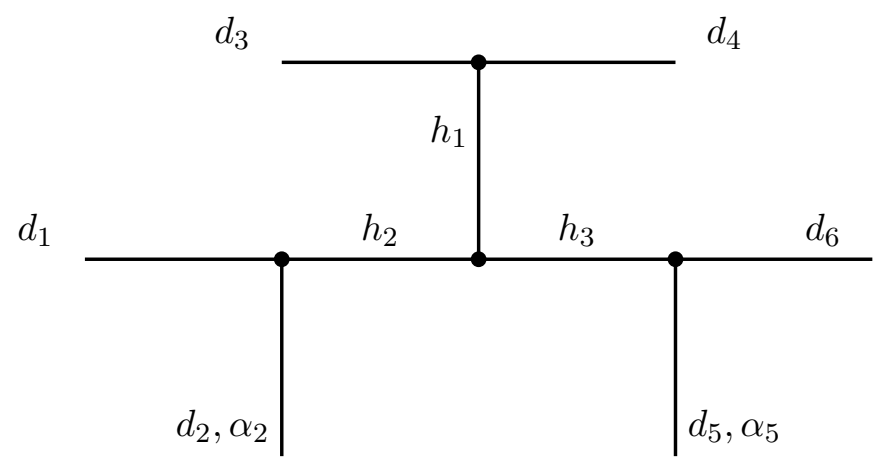

Figure 4. 6-point conformal block in the non-linear channel.

were the coefficients

$$
\begin{aligned}
& R_{r, s}^{1}\left(h_{1}\right)= J_{r, s}\left(h_{1}\right) A_{r, s}\left(h_{1}\right) S_{r s}^{\alpha_{2}} \sigma_{r, s}^{\alpha_{2}}\left(d_{2}, d_{1} ; h_{1}\right) \sigma_{r, s}^{\alpha_{3}}\left(d_{3}, h_{2} ; h_{1}\right), \\
& R_{r, s}^{i}\left(h_{i}\right)= J_{r, s}\left(h_{i}\right) A_{r, s}\left(h_{i}\right) S_{r s}^{\alpha_{i+2}} \sigma_{r, s}^{\alpha_{i+2}}\left(d_{i+2}, h_{i-1} ; h_{i}\right) \sigma_{r, s}^{\alpha_{i+3}}\left(d_{i+3}, h_{i+1} ; h_{i}\right), \\
& 1<i<N-3, \\
& R_{r, s}^{N-3}\left(h_{N-3}\right)= J_{r, s}\left(h_{N-3}\right) A_{r, s}\left(h_{N-3}\right) S_{r s}^{\alpha_{N-2}} \sigma_{r, s}^{\alpha_{N-2}}\left(d_{N-2}, h_{N-4} ; h_{N-3}\right) \\
& \times \sigma_{r, s}^{\alpha_{N-1}}\left(d_{N-1}, d_{N} ; h_{N-3}\right)
\end{aligned}
$$

and $G_{n_{1}, \ldots, n_{N-3}}$ is constructed from the matrix elements listed in appendix A.

\subsection{Non-linear channel blocks}

In this subsection we consider a particular example of non-linear block. We fix the OPE channel according to the diagram in figure 4.

The block reads

$$
\begin{aligned}
F= & \sum_{\vec{k}_{i}, \vec{p}_{i}} q_{1}^{p_{1}} q_{2}^{p_{2}} q_{3}^{p_{3}} B^{\vec{k}_{1}, \vec{p}_{1}}\left(h_{1}\right) B^{\vec{k}_{2}, \vec{p}_{2}}\left(h_{2}\right) B^{\vec{k}_{3}, \vec{p}_{3}}\left(h_{3}\right) \times \\
& \times \rho\left(\nu_{3}, \mathcal{L}_{\vec{k}_{1}} \nu_{h_{1}}, \nu_{4}\right) \rho\left(\mathcal{L}_{\vec{p}_{2}} \nu_{h_{2}}, \mathcal{L}_{\vec{p}_{1}} \nu_{h_{1}}, \mathcal{L}_{\vec{p}_{3}} \nu_{h_{3}}\right) \rho^{\alpha_{2}}\left(\nu_{1}, \nu_{2}, \mathcal{L}_{\vec{k}_{2}} \nu_{h_{2}}\right) \rho^{\alpha_{5}}\left(\mathcal{L}_{\vec{k}_{3}} \nu_{h_{3}}, \nu_{6}, \nu_{5}\right),
\end{aligned}
$$

where the sum goes over the set of half-integer partitions. We fix operators $\nu_{1}, \nu_{2}, \nu_{6}$ to be located at $\infty, 1,0$ respectively, and, as explained in appendix C (see figure 8), the moduli of the punctured Riemann sphere are expressed in terms of the positions of the remaining operators as follows: $q_{1}=1-z_{4}, q_{2}=\frac{1}{z_{3}}, q_{3}=z_{5}$. Exploiting the pole decomposition in 


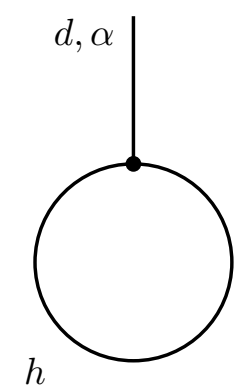

Figure 5. Torus 1-point conformal block.

each intermediate channel, we get the following recursion

$$
\begin{aligned}
F= & G+\sum_{\substack{r \geq 2, s \geq 1 \\
r+s \in 2 \mathbb{N}}} \frac{q_{1}^{\frac{r s}{2}} R_{r, s}^{1}}{c-c_{r, s}\left(h_{1}\right)} F\left(h_{1} \rightarrow h_{1}+\frac{r s}{2}, c \rightarrow c_{r, s}\left(h_{1}\right)\right) \\
& +\sum_{\substack{r \geq 2, s \geq 1 \\
r+s \in 2 \mathbb{N}}} \frac{q_{2}^{\frac{r s}{2}} R_{r, s}^{2}}{c-c_{r, s}\left(h_{2}\right)} F\left(h_{2} \rightarrow h_{2}+\frac{r s}{2}, c \rightarrow c_{r, s}\left(h_{2}\right)\right) \\
& +\sum_{\substack{r \geq 2, s \geq 1 \\
r+s \in 2 \mathbb{N}}} \frac{q_{3}^{\frac{r s}{2}} R_{r, s}^{3}}{c-c_{r, s}\left(h_{3}\right)} F\left(h_{3} \rightarrow h_{3}+\frac{r s}{2}, c \rightarrow c_{r, s}\left(h_{3}\right)\right),
\end{aligned}
$$

where

$$
\begin{aligned}
& R_{r, s}^{1}=A_{r, s}\left(h_{1}\right) J_{r, s}\left(h_{1}\right) \sigma_{r, s}^{\alpha_{4}}\left(d_{3}, d_{4} ; h_{1}\right) \sigma_{r, s}^{0}\left(h_{2}, h_{3} ; h_{1}\right) \\
& R_{r, s}^{2}=A_{r, s}\left(h_{2}\right) J_{r, s}\left(h_{2}\right) S_{r, s}^{\alpha_{2}} \sigma_{r, s}^{\alpha_{2}}\left(d_{1}, d_{2} ; h_{2}\right) \sigma_{r, s}^{0}\left(h_{3}, h_{1} ; h_{2}\right), \\
& R_{r, s}^{3}=A_{r, s}\left(h_{3}\right) J_{r, s}\left(h_{3}\right) \sigma_{r, s}^{\alpha_{5}}\left(d_{5}, d_{6} ; h_{3}\right) \sigma_{r, s}^{0}\left(h_{1}, h_{2} ; h_{3}\right) .
\end{aligned}
$$

One can compare these results to the AGT construction for the non-linear Virasoro block found in [43].

\section{Blocks on the torus}

\subsection{Previous results: 1-point blocks}

Here we apply the recursive approach to computing NS 1-point conformal blocks on the torus. The corresponding graph is represented in figure 5 .

By definition, the block is given by

$$
\begin{aligned}
F & =\sum_{n \in \frac{1}{2} \mathbb{N}_{0}}^{\infty} F_{n}, \\
F_{n} & =\sum_{k=n} B_{n}^{\vec{k}, \vec{k}}(h) \rho^{\alpha}\left(\mathcal{L}_{\vec{k}} \nu_{h}, \nu_{d}, \mathcal{L}_{\vec{k}} \nu_{h}\right),
\end{aligned}
$$

where $q=e^{2 \pi i \tau}$ and $\tau$ is the modular parameter of the torus (see appendix C, figure 9). 
The residue of the conformal block is given by

$$
\lim _{h \rightarrow d_{r, s}}\left(h-d_{r, s}\right) F=q^{\frac{r s}{2}} A_{r, s}(c) \sum_{k \in \frac{1}{2} \mathbb{N}_{0}} z^{k} B^{\vec{k}, \vec{k}}\left(d_{r, s}+\frac{r s}{2}\right) \mu_{r, s}^{\alpha}(d ; c) \rho^{\alpha}\left(\mathcal{L}_{\vec{k}} \nu_{d_{r, s}+\frac{r s}{2}}, \nu_{d}, \mathcal{L}_{\vec{k}} \nu_{d_{r, s}+\frac{r s}{2}}\right) \text {. }
$$

After changing the variables one gets

$$
\begin{aligned}
\lim _{c \rightarrow c_{r, s}(h)}\left(c-c_{r, s}(h)\right) F= & q^{\frac{r s}{2}} J_{r, s}(h) A_{r, s}(h) q^{\frac{r s}{2}} \\
& \times \sum_{k \in \frac{1}{2} \mathbb{N}_{0}} z^{k} B^{\vec{k}, \vec{k}}\left(d_{r, s}+\frac{r s}{2}\right) \mu_{r, s}^{\alpha}(d ; h) \rho^{\alpha}\left(\mathcal{L}_{\vec{k}} \nu_{d_{r, s}+\frac{r s}{2}}, \nu_{d}, \mathcal{L}_{\vec{k}} \nu_{d_{r, s}+\frac{r s}{2}}\right) .
\end{aligned}
$$

This defines the singular part of the pole decomposition. Now, to get $c$-recursion relations, it remains to find the light asymptotic of the block. In the full analogy with the Virasoro case [44], where the light 1-point block on the torus factorizes into the vacuum character and the global block, the NS light block splits into the NS vacuum character and the global block of $\operatorname{osp}(1 \mid 2)$ algebra [45]

$$
G=\prod_{n=2}^{\infty} \frac{1+q^{n-\frac{1}{2}}}{1-q^{n}} \times\left. F\right|_{o s p(1 \mid 2)}=\sum_{n \in \frac{1}{2} \aleph_{0}}^{\infty} G_{n} q^{n} .
$$

The global block reads

$$
\left.F\right|_{o s p(1 \mid 2)}=\sum_{n \in \mathbb{N}_{0}} \sum_{\beta \in\{0,1\}} q^{n+\frac{\beta}{2}} \frac{\rho^{\beta, \alpha, \beta}\left(L_{-1}^{n} \nu_{h}, \nu_{d}, L_{-1}^{n} \nu_{h}\right)}{\rho^{\beta, 0, \beta}\left(L_{-1}^{n} \nu_{h}, \mathbb{1}, L_{-1}^{n} \nu_{h}\right\rangle}=\sum_{n \in \mathbb{N}_{0}} \sum_{\beta \in\{0,1\}} q^{n+\frac{\beta}{2}} \frac{\tau_{n, n}^{\beta, \alpha, \beta}(h, d, h)}{n !(2 h)_{n+\beta}},
$$

where the explicit form of the matrix elements is given in appendix A. Finally, we get the following recursion relations for the one-point torus superblock coefficients

$$
F_{n}=G_{n}+\sum_{\substack{r \geq 2, s \geq 1 \\ r+s \in 2 \mathrm{~N}}}^{r s \leq 2 n} \frac{R_{r, s}(h)}{c-c_{r, s}(h)} F_{n-\frac{r s}{2}}\left(h \rightarrow h+\frac{r s}{2}, c \rightarrow c_{r, s}\right)
$$

where the coefficients $G_{n}$ are defined in (4.4), (4.5) and the residue coefficients are

$$
R_{r, s}(h)=J_{r, s}(h) A_{r, s}(h) \mu_{r, s}^{\alpha}(d ; h) .
$$

The lower coefficients of the block $F$ are collected in appendix B.

\section{2 $\quad N$-point blocks in the necklace channel}

The generalization of the previous consideration on the $N$-point case is pretty straightforward. We define the block

$$
\begin{aligned}
F= & \sum_{\vec{k}_{i}, \vec{p}_{i}} q_{1}^{k_{1}} \ldots q_{N}^{k_{N}} B^{\vec{k}_{1}, \vec{p}_{1}}\left(h_{1}\right) \ldots B^{\vec{k}_{N}, \vec{p}_{N}}\left(h_{N}\right) \rho^{\alpha_{1}}\left(L_{\vec{p}_{1}} \nu_{1}, \nu_{h_{1}}, L_{\vec{k}_{2}} \nu_{2}\right) \ldots \\
& \times \rho^{\alpha_{N}}\left(L_{\vec{p}_{N}} \nu_{N}, \nu_{h_{N}}, L_{\vec{k}_{1}} \nu_{1}\right),
\end{aligned}
$$

corresponding to the diagram in figure 6 (for the definition of $q_{i}$, see appendix $\mathrm{C}$, figure 10). 


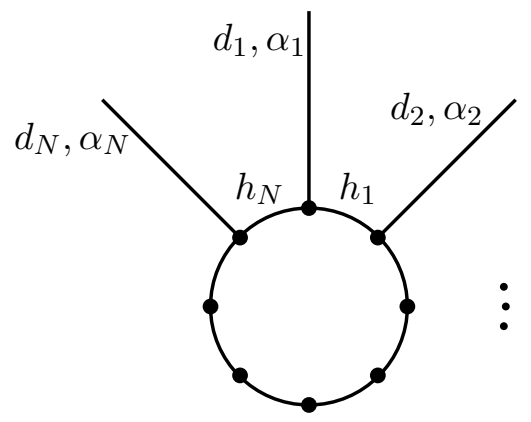

Figure 6. Torus $N$-point conformal block in the necklace channel.

Using our general scheme, we get the following decomposition

$$
F=G+\sum_{i=1}^{N} \sum_{\substack{r \geq 2, s \geq 1, r_{i}+s_{i} \in 2 \mathfrak{N}}} \frac{R_{r_{i}, s_{i}}^{i}}{c-c_{r_{i}, s_{i}}\left(h_{i}\right)} F\left(c \rightarrow c_{r_{i}, s_{i}}\left(h_{i}\right), h_{i} \rightarrow h_{i}+\frac{r_{i} s_{i}}{2}\right)
$$

where

$$
R^{1}=J_{r, s}\left(h_{1}\right) A_{r, s}\left(h_{1}\right) S_{r s}^{\alpha_{1}} \sigma_{r, s}^{\alpha_{1}}\left(d_{1}, h_{N} ; h_{1}\right) \sigma_{r, s}^{\alpha_{2}}\left(d_{2}, h_{2} ; h_{1}\right)
$$

and other $R$-coefficients are obtained by replacing cyclically $h_{i}, d_{i}$ and $\alpha_{i}$. The light block is

$$
G=\prod_{n_{1}=2}^{\infty} \ldots \prod_{n_{N}=2}^{\infty} \frac{1+q_{1}^{n_{1}-\frac{1}{2}} \ldots q_{N}^{n_{N}-\frac{1}{2}}}{1-q_{1}^{n_{1}} \ldots q_{N}^{n_{N}}} \times\left. F\right|_{o s p(1 \mid 2)}=\sum_{n_{i} \in \frac{1}{2} \mathbb{N}_{0}} G_{n_{1} \ldots n_{N}} q_{1}^{n_{1}} \ldots q_{N}^{n_{N}}
$$

with the global block constructed of the $\operatorname{osp}(1 \mid 2)$ matrix elements (see appendix A)

$$
\left.F\right|_{o s p(1 \mid 2)}=\sum_{n_{i} \in \mathbb{N}_{0}} \sum_{\beta_{i} \in\{0,1\}} \frac{\tau^{\beta_{1}, \alpha_{2}, \beta_{1}}\left(h_{1}, d_{2}, h_{2}\right)}{\left(2 h_{1}\right)_{n_{1}+\beta_{1}} n_{1} !} \ldots \frac{\tau^{\beta_{N}, \alpha_{1}, \beta_{N}}\left(h_{N}, d_{1}, h_{1}\right)}{\left(2 h_{N}\right)_{n_{N}+\beta_{N}} n_{N} !} q_{1}^{n_{1}+\beta_{1}} \ldots q_{N}^{n_{N}+\beta_{N}} .
$$

\section{Solutions of the recursion relations}

Solution for 4-point blocks on the sphere. Here we describe an approach to the computation of the superconformal blocks, based on the obtained $c$-recursion relations. The main idea of [38], where an analogues approach has been proposed for Virasoro blocks, was to rearrange the OPE in a given intermediate channel in order to obtain the sum over modules growing from quasiprimary states (annihilated by $L_{1}$ ). In our case the sum over the given NS module splits into the sum over $\operatorname{ssp}(1 \mid 2)$ modules, growing from superquasiprimary states (annihilated by $L_{1}, G_{\frac{1}{2}}$, see appendix A). For 4-point NS blocks it leads to the following decomposition

$$
F=\sum_{p \in \frac{1}{2} \mathbb{N}_{0}} z^{p} \chi_{p} G(h \rightarrow h+p)
$$


where $p$ runs over levels of the quasiprimaries and $G$ is the light block (3.9). Note that in this decomposition $\chi_{0}=1, \chi_{\frac{1}{2}}=0, \chi_{1}=0$, because there are no quasiprimary states on the levels $\frac{1}{2}$ and 1 , if $h$ is not degenerate. The general coefficients $\chi_{p}$ can be found from the requirement that the ansatz (5.1) satisfies $c$-recursion constraints (3.10). We get the following expression

$$
\chi_{p}=\sum_{j=1}^{\left[\frac{p}{2}\right]} \prod_{\ell=1}^{j} \sum_{\substack{r_{\ell} \geq 2, s_{\ell} \geq 1}}^{\infty} \gamma_{r_{\ell}, s_{\ell}}\left(c_{\mathrm{eff}}^{(\ell)}, d_{i}, h_{\mathrm{eff}}^{(\ell)}\right), \text { with } \sum_{\ell=1}^{j} r_{\ell} s_{\ell}=2 p \text { and } r_{i}+s_{i} \in 2 \mathbb{N}
$$

and

$$
\gamma_{r, s}\left(c, d_{i}, h\right):=\frac{R_{r, s}(h)}{c-c_{r, s}(h)},
$$

where $R_{r, s}$ are given in (3.11) and the effective parameters are:

$$
\begin{aligned}
h_{\mathrm{eff}}^{(\ell)} & :=h+\Delta h^{(\ell-1)}, \\
\Delta h^{(\ell)} & :=\sum_{r=1}^{l} \frac{r_{\ell} s_{\ell}}{2}, \\
c_{\mathrm{eff}}^{(\ell)} & :=c_{r_{\ell-1}, s_{\ell-1}}\left(h_{\mathrm{eff}}^{(\ell-1)}\right) .
\end{aligned}
$$

These relations allow to compute conformal blocks recursively. We note that the corresponding iteration procedure is more appropriate, as compared to the standard c-recursion, for numerical computations of the conformal blocks. ${ }^{8}$ In particular, in this version at each level there is no need to keep analytic expressions for the lower levels' coefficients, unlike the original $c$-recursion procedure.

Solution for 1-point blocks on the torus. In the same way as we obtain (5.1) for the spherical 4-point case, we find the solution of the $c$-recursion (4.6) on the torus:

$$
F=\sum_{p \in \frac{1}{2} \mathbb{N}_{0}} q^{p} \chi_{p} G(h \rightarrow h+p)
$$

where $F$ is the torus 1-point block (4.1) and $G$ is the torus light block (4.4). The coefficients are the following

$$
\begin{aligned}
\chi_{p} & =\sum_{j=1}^{\left[\frac{p}{2}\right]} \prod_{\substack{\ell=1 \\
r_{\ell} \geq 2, s_{\ell} \geq 1}}^{j} \sum_{r_{\ell}, s_{\ell}}\left(c_{\mathrm{eff}}^{(\ell)}, d, h_{\mathrm{eff}}^{(\ell)}\right), \text { with } \sum_{\ell=1}^{j} r_{\ell} s_{\ell}=2 p \text { and } r_{i}+s_{i} \in 2 \mathbb{N}, \\
\gamma_{r, s}(c, d, h) & :=\frac{R_{r, s}}{c-c_{r, s}(h)},
\end{aligned}
$$

where $R_{r, s}$ are given in (4.7) and the effective parameters are defined in (5.4). The generalization of this construction to the torus $N$-point block is straightforward.

\footnotetext{
${ }^{8}$ For the rational values of the central charge $c$, corresponding to minimal models, the numerical recursion requires certain modification, see, e.g. [46].
} 


\section{Discussion}

In this paper we have analyzed the Neveu-Schwarz sector of the $\mathcal{N}=1$ super-Virasoro CFT. We obtained $c$-recursion relations for multi-point superconformal blocks on the sphere and on the torus, involving upper and lower components of primary supermultiplets, which are required for constructing multi-point correlation functions in $\mathcal{N}=1$ CFT minimal models, as well as in the $\mathcal{N}=1$ supersymmetric Liouville filed theory. Similarly to the "standard" four-point (super-)Virasoro case, the multi-point $c$-recursion is based on the analysis of the analytic structure, which is characterized by two main ingredients: the singular and the regular parts.

The singular part, which is defined by OPE, is obtained by analyzing superconformal fusion rules. The key point here is that in the multi-point supersymmetric case the singular part still contains only the contribution of simple poles the $\left(c-c_{r, s}\right)^{-1}$ and the residues are expressed in a simple manner in terms of the sypersymmetric fusion polynomials. The regular part is governed by the light asymptotic, which can be expressed in terms of global blocks of $\operatorname{sp}(1 \mid 2)$ algebra and NS vacuum characters. Rather simple representation theory allows to find explicitly the $\operatorname{osp}(1 \mid 2)$ matrix elements and to reduce the computation of the light blocks (in general topology) to the problem of identification of the effective plumbing parameterization of the moduli space.

It is shown that the recursion relations can be effectively rewritten in terms of the light blocks with shifted values of the intermediate conformal dimension parameters, which allows to significantly simplify recursion formulas and makes them more suitable for numeric computations.

There are several possible extensions of our results. A natural extension is to analyze the Ramond sector of $\mathcal{N}=1$ superconformal theory. The careful analysis of higher genus cases and, in particular, of the genus-two case is desirable (see, e.g., [42, 47-49]).

\section{Acknowledgments}

We would like to thank M. Bershtein for useful comments. The research was supported by Foundation for the Advancement of Theoretical Physics and Mathematics "Basis". The work of R.G. has been funded by the Russian Academic Excellence Project '5-100'. This research was supported in part by the International Center for Theoretical Sciences (ICTS) during a visit for participating in the program - Kavli Asian Winter School (KAWS) on Strings, Particles and Cosmology 2018.

\section{A General $\operatorname{osp}(1 \mid 2)$ matrix elements}

Here we work with the global part of NS superalgebra only, that is $\operatorname{ssp}(1 \mid 2)$.

$$
\begin{aligned}
{\left[L_{n}, L_{m}\right] } & =(n-m) L_{m+n}, \\
{\left[L_{n}, G_{k}\right] } & =\frac{n-2 k}{2} G_{n+k}, \\
\left\{G_{k}, G_{l}\right\} & =2 L_{k+l},
\end{aligned}
$$


where $m, n=-1,0,1$ and $k, l=-\frac{1}{2}, \frac{1}{2}$. The $\operatorname{osp}(1 \mid 2)$ highest weight is defined by

$$
L_{1}|d\rangle=0, \quad G_{\frac{1}{2}}|d\rangle=0
$$

We use the following identities

$$
\begin{aligned}
L_{1} L_{-1}^{m}|d\rangle & =(2 d+m-1) L_{-1}^{m-1}|d\rangle, \\
G_{\frac{1}{2}} L_{-1}^{m}|d\rangle & =m G_{-\frac{1}{2}} L_{-1}^{m-1}|d\rangle, \\
G_{\frac{1}{2}} G_{-\frac{1}{2}} L_{-1}^{m}|d\rangle & =(2 d+m) L_{-1}^{m}|d\rangle, \\
L_{1} G_{-\frac{1}{2}} L_{-1}^{m}|d\rangle & =(2 d+2 m-1) G_{-\frac{1}{2}} L_{-1}^{m-1}|d\rangle,
\end{aligned}
$$

to derive the full set of global matrix elements. We set the following notation

$$
\rho\left(G_{-\frac{1}{2}}^{\alpha_{1}} L_{-1}^{k} \nu_{1}, G_{-\frac{1}{2}}^{\alpha_{2}} \nu_{2}, G_{-\frac{1}{2}}^{\alpha_{3}} L_{-1}^{m} \nu_{3}\right)=\tau_{k, m}^{\alpha_{1}, \alpha_{2}, \alpha_{3}}\left(d_{1}, d_{2}, d_{3}\right)
$$

where the $\tau_{k, m}^{\alpha_{1}, \alpha_{2}, \alpha_{3}}$ are given by

$$
\begin{aligned}
\tau_{k, m}^{0,0,0}\left(d_{1}, d_{2}, d_{3}\right)= & \sum_{p=0}^{\min [k, m]} \frac{k !}{p !(k-p) !}\left(2 d_{3}+m-1\right)^{(p)} m^{(p)} \\
& \times\left(d_{2}+d_{3}-d_{1}\right)_{m-p}\left(d_{1}+d_{2}-d_{3}+p-m\right)_{k-p} \\
\tau_{k, m}^{1,0,0}\left(d_{1}, d_{2}, d_{3}\right)= & \sum_{p=0}^{\min [k, m]} \frac{m !}{p !(m-p) !}\left(2 d_{3}+m\right)^{(p)} k^{(p)} \\
& \times\left(d_{2}+d_{3}-d_{1}+\frac{1}{2}\right)_{m-p}\left(d_{1}+d_{2}-d_{3}+\frac{1}{2}-m+p\right)_{k-p}, \\
\tau_{k, m}^{0,1,0}\left(d_{1}, d_{2}, d_{3}\right)= & \sum_{p=0}^{\min [k, m]} \frac{k !}{p !(k-p) !}\left(2 d_{3}+m-1\right)^{(p)} m^{(p)} \\
& \times\left(d_{2}+d_{3}-d_{1}+\frac{1}{2}\right)_{m-p}\left(d_{1}+d_{2}-d_{3}+p-m+\frac{1}{2}\right)_{k-p}, \\
\tau_{k, m}^{0,0,1}\left(d_{1}, d_{2}, d_{3}\right)= & \sum_{p=0}^{\min [k, m]} \frac{k !}{p !(k-p) !}\left(2 d_{3}+m\right)^{(p)} m^{(p)} \\
& \times\left(d_{2}+d_{3}-d_{1}+\frac{1}{2}\right)_{m-p}\left(d_{1}+d_{2}-d_{3}-\frac{1}{2}+p-m\right)_{k-p}, \\
& \sum_{p=0}^{\min [k, m]} \frac{k !}{p !(k-p) !}\left(2 d_{3}+m-1\right)^{(p)} m^{(p)} \\
& \times\left(d_{2}+d_{3}-d_{1}\right){ }_{m-p}\left(d_{1}+d_{2}-d_{3}+p-m+1\right)_{k-p}\left(d_{1}+d_{2}-d_{3}\right), \\
& \sum_{k, m}^{1,1,0}\left(d_{1}, d_{2}, d_{3}\right)= \\
\tau_{k, m}^{1,0,1}\left(d_{1}, d_{2}, d_{3}\right)= & \sum_{p=0}^{\min [k, m]} \frac{k !}{p !(k-p) !}\left(2 d_{3}+m\right)^{(p)} m^{(p)} \\
& \left.\times d_{3}-d_{1}\right)_{m-p}\left(d_{1}+d_{2}-d_{3}+p-m\right)_{k-p}\left(d_{1}-d_{2}+d_{3}\right),
\end{aligned}
$$




$$
\begin{aligned}
\tau_{k, m}^{0,1,1}\left(d_{1}, d_{2}, d_{3}\right)= & -\sum_{p=0}^{\min [k, m]} \frac{k !}{p !(k-p) !}\left(2 d_{3}+m\right)^{(p)} m^{(p)} \\
& \times\left(d_{2}+d_{3}-d_{1}\right)_{m-p+1}\left(d_{1}+d_{2}-d_{3}+p-m\right)_{k-p}, \\
\tau_{k, m}^{1,1,1}\left(d_{1}, d_{2}, d_{3}\right)= & \sum_{p=0}^{\min [k, m]} \frac{k !}{p !(k-p) !}\left(2 d_{3}+m\right)^{(p)} m^{(p)}\left(d_{2}+d_{3}-d_{1}+\frac{1}{2}\right)_{m-p} \\
& \times\left(d_{1}+d_{2}-d_{3}+p-m+\frac{1}{2}\right)_{k-p}\left(d_{1}+d_{2}+d_{3}+\frac{1}{2}\right),
\end{aligned}
$$

where $(x)^{(m)}$ and $(x)_{(m)}$ are falling and rising Pochhammer symbols respectively. Not all of these elements are independent due to the reflection properties (2.22). The most general $\operatorname{osp}(1 \mid 2)$ matrix element reads

$$
\begin{aligned}
& \rho\left(G_{-\frac{1}{2}}^{\alpha_{1}} L_{-1}^{k} \nu_{1}, G_{-\frac{1}{2}}^{\alpha_{2}} L_{-1}^{m} \nu_{2}, G_{-\frac{1}{2}}^{\alpha_{3}} L_{-1}^{n} \nu_{3}\right) \\
& \quad=\left(d_{1}-d_{2}-d_{3}+\frac{\alpha_{1}-\alpha_{2}-\alpha_{3}}{2}+k-m-n\right)^{(m)} \tau_{k, n}^{\alpha_{1}, \alpha_{2}, \alpha_{3}}\left(d_{1}, d_{2}, d_{3}\right) .
\end{aligned}
$$

\section{B Some explicit coefficients}

Here we provide the first few coefficients of the torus conformal block with the lower component of the vertex operator (4.1):

$$
\begin{array}{r}
F_{0}=1, \quad F_{1 / 2}=\frac{\rho\left(G_{-\frac{1}{2}} \nu_{h}, \nu_{d}, G_{-\frac{1}{2}} \nu_{h}\right)}{\left\langle G_{-\frac{1}{2}} \nu_{h} \mid G_{-\frac{1}{2}} \nu_{h}\right\rangle}=\frac{1}{2 h}(2 h-d), \\
F_{1}=\frac{\rho\left(L_{-1} \nu_{h}, \nu_{d}, L_{-1} \nu_{h}\right)}{\left\langle L_{-1} \nu_{h} \mid L_{-1} \nu_{h}\right\rangle}=\frac{2 h+d(d-1)}{2 h}
\end{array}
$$

On the level $\frac{3}{2}$ we fix the following ordering: $\left\{G_{-\frac{1}{2}} L_{-1}, G_{-3 / 2}\right\}$. The $\frac{3}{2}$-level coefficient of the conformal block is

$$
F_{\frac{3}{2}}=\operatorname{Tr}\left(B_{\frac{3}{2}}^{-1}\left(B_{\frac{3}{2}}+T_{\frac{3}{2}}\right)\right)
$$

where

$$
B_{\frac{3}{2}}=\left(\begin{array}{cc}
2 h(2 h+1) & 4 h \\
4 h & 2 h+c
\end{array}\right), \quad T_{\frac{3}{2}}=\left(\begin{array}{cc}
\tau_{1,1}(\tilde{h}, d, \tilde{h}) & -d(d+1) \\
-d(d+1) & -3 d
\end{array}\right) .
$$

On the level 2 two we fix the following ordering: $\left\{L_{-1}^{2}, L_{-2}, G_{-\frac{1}{2}} G_{-3 / 2}\right\}$. The second level coefficient of the conformal block is

$$
F_{2}=\operatorname{Tr}\left(B_{2}^{-1}\left(B_{2}+T_{2}\right)\right)
$$

where

$$
\begin{aligned}
B_{2} & =\left(\begin{array}{ccc}
4 h(2 h+1) & 6 h & 8 h \\
6 h & 4 h+\frac{3 c}{4} & 3 h+\frac{3 c}{2} \\
8 h & 3 h+\frac{3 c}{2} c(3+2 h)+2 h(2 h-1)
\end{array}\right), \\
T_{2} & =\left(\begin{array}{ccc}
d(d-1)(8 h+d(d-1)+2) & 2 d\left(d^{2}-1\right) & d(d-1)(2+3 d) \\
2 d\left(d^{2}-1\right) & 4 d(d-1) & 6 d(d-1) \\
d(d-1)(2+3 d) & 6 d(d-1) & d(11 d-8 h-9-c)
\end{array}\right) .
\end{aligned}
$$




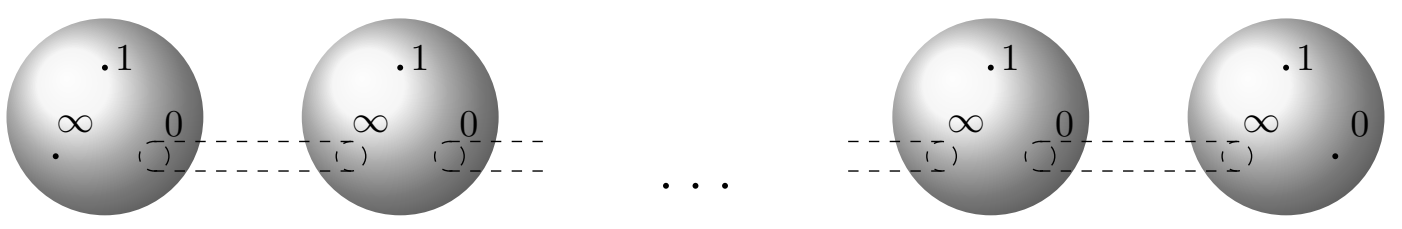

Figure 7. The plumbing construction for the $N$-punctured sphere.

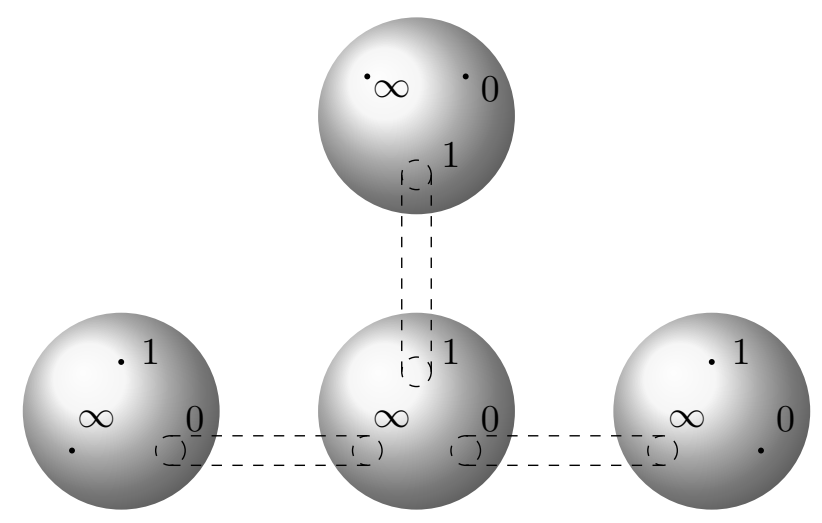

Figure 8. The plumbing construction for the 6-point non-linear block.

\section{Plumbing constructions}

For completeness, we describe here the relation (mostly borrowed from the bosonic case [1]) between the local parametrization of the moduli space and the elements of the associated dual diagram. We begin with the plumbing construction associated with figure 1. Accordingly, building blocks are two two-punctured and one-holed spheres. We fix the first sphere to have punctures at 0 and 1 and a hole at $\infty$. The second sphere has punctures at 1 and $\infty$ and a hole at 0 . We glue these spheres together by their boundaries via $\operatorname{SL}(2, \mathbb{C})$ map. Let us choose the coordinates on the spheres to be $w_{1}$ and $w_{2}$, then, the gluing map is $w_{2}=z w_{1}$. Thus, we have the sphere with four punctures at $0,1, z, \infty$. The diagram corresponds to the case of $\nu_{4}, \nu_{3}, \nu_{2}, \nu_{1}$ located at these punctures respectively.

We proceed with the case represented in figure 3. We have $N-2$ spheres: two twopunctured and one-holed spheres and $N-4$ two-holed and one-punctured spheres. The gluing of the holes is depicted in figure 7 .

We introduce coordinates $w_{1}, \ldots, w_{N-2}$ on the spheres and the gluing maps $w_{i}=$ $q_{i} w_{i+1}$, where $1 \leq i \leq N-3$. We get the sphere with $N$ punctures at

$$
0,1, q_{1}, q_{1} q_{2}, \ldots, q_{1} \ldots q_{N-3}, \infty
$$

Inserting $\nu_{N}, \nu_{N-1}, \ldots, \nu_{1}$ at these punctures respectively we come to (3.18).

Now we consider 6-point block with the dual diagram in figure 4. The corresponding plumbing graph is shown in figure 8. It consists of three two-punctured and one-holed spheres and one three-holed sphere. 


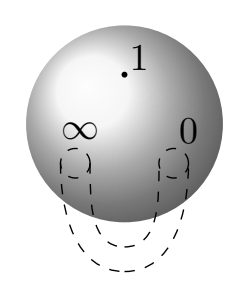

Figure 9. The plumbing construction for the 1-point torus block.

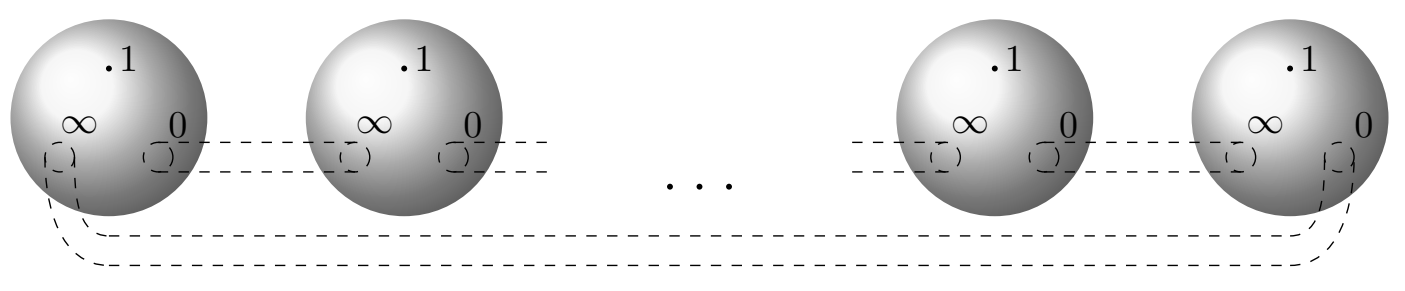

Figure 10. The plumbing construction for the $N$-punctured torus.

Introducing coordinates $w_{i}, i=1,2,3$ on two-punctured spheres numerated from left to right and $w_{4}$ on the three-punctured sphere in the middle one, we get the following maps

$$
w_{1}=q_{2} w_{4}, \quad w_{2}=1+\frac{q_{1}}{w_{4}-1}, \quad w_{3}=\frac{w_{4}}{q_{3}} .
$$

Gluing via these mappings we get the sphere with 6 punctures located at points:

$0,1-q_{1}, \frac{1}{q_{2}}, q_{3}, 1, \infty$.

For the torus one-point block (figure 5) we have the plumbing construction in figure 9.

One-punctured torus is obtained from two-holed one-punctured sphere by identification $w \sim q w$ and $q$ is the modulus of the given torus.

The dual diagram for the torus $N$-point block in the necklace channel (figure 6 ) corresponds to the plumbing construction depicted in figure 10 .

We obtain the torus with modulus $q=q_{1} \ldots q_{N}$ and punctures located at $1, q_{1}, q_{1} q_{2}, \ldots, q_{1} \ldots q_{N-1}$.

Open Access. This article is distributed under the terms of the Creative Commons Attribution License (CC-BY 4.0), which permits any use, distribution and reproduction in any medium, provided the original author(s) and source are credited.

\section{References}

[1] M. Cho, S. Collier and X. Yin, Recursive Representations of Arbitrary Virasoro Conformal Blocks, arXiv:1703.09805 [INSPIRE].

[2] A.A. Belavin, A.M. Polyakov and A.B. Zamolodchikov, Infinite Conformal Symmetry in Two-Dimensional Quantum Field Theory, Nucl. Phys. B 241 (1984) 333 [INSPIRE].

[3] D. Poland, S. Rychkov and A. Vichi, The Conformal Bootstrap: Theory, Numerical Techniques and Applications, arXiv:1805.04405 [INSPIRE]. 
[4] L.F. Alday, D. Gaiotto and Y. Tachikawa, Liouville Correlation Functions from Four-dimensional Gauge Theories, Lett. Math. Phys. 91 (2010) 167 [arXiv:0906.3219] [INSPIRE].

[5] V. Belavin and B. Feigin, Super Liouville conformal blocks from $N=2 \mathrm{SU}(2)$ quiver gauge theories, JHEP 07 (2011) 079 [arXiv:1105.5800] [INSPIRE].

[6] V.A. Alba, V.A. Fateev, A.V. Litvinov and G.M. Tarnopolskiy, On combinatorial expansion of the conformal blocks arising from AGT conjecture, Lett. Math. Phys. 98 (2011) 33 [arXiv: 1012.1312] [INSPIRE].

[7] A. Belavin and V. Belavin, AGT conjecture and Integrable structure of Conformal field theory for $c=1$, Nucl. Phys. B 850 (2011) 199 [arXiv:1102.0343] [INSPIRE].

[8] A.B. Zamolodchikov, Conformal symmetry in two-dimensions: an explicit recurrence formula for the conformal partial wave amplitude, Commun. Math. Phys. 96 (1984) 419 [INSPIRE].

[9] A.B. Zamolodchikov, Conformal symmetry in two-dimensional space: Recursion representation of conformal block, Theor. Math. Phys. 73 (1987) 1088.

[10] A.B. Zamolodchikov and A.B. Zamolodchikov, Structure constants and conformal bootstrap in Liouville field theory, Nucl. Phys. B 477 (1996) 577 [hep-th/9506136] [INSPIRE].

[11] L. Hadasz, Z. Jaskolski and P. Suchanek, Recursion representation of the Neveu-Schwarz superconformal block, JHEP 03 (2007) 032 [hep-th/0611266] [INSPIRE].

[12] V.A. Belavin, $N=1$ supersymmetric conformal block recursion relations, Theor. Math. Phys. 152 (2007) 1275 [Teor. Mat. Fiz. 152 (2007) 476] [hep-th/0611295] [INSPIRE].

[13] A. Belavin, V. Belavin, A. Neveu and A. Zamolodchikov, Bootstrap in Supersymmetric Liouville Field Theory. I. NS Sector, Nucl. Phys. B 784 (2007) 202 [hep-th/0703084] [INSPIRE].

[14] L. Hadasz, Z. Jaskolski and P. Suchanek, Recursive representation of the torus 1-point conformal block, JHEP 01 (2010) 063 [arXiv:0911.2353] [INSPIRE].

[15] L. Hadasz, Z. Jaskolski and P. Suchanek, Recurrence relations for toric $N=1$ superconformal blocks, JHEP 09 (2012) 122 [arXiv:1207.5740] [INSPIRE].

[16] T. Hartman, Entanglement Entropy at Large Central Charge, arXiv:1303.6955 [INSPIRE].

[17] C.T. Asplund, A. Bernamonti, F. Galli and T. Hartman, Holographic Entanglement Entropy from 2d CFT: Heavy States and Local Quenches, JHEP 02 (2015) 171 [arXiv:1410.1392] [INSPIRE].

[18] A.L. Fitzpatrick, J. Kaplan and M.T. Walters, Universality of Long-Distance AdS Physics from the CFT Bootstrap, JHEP 08 (2014) 145 [arXiv:1403.6829] [INSPIRE].

[19] E. Hijano, P. Kraus and R. Snively, Worldline approach to semi-classical conformal blocks, JHEP 07 (2015) 131 [arXiv: 1501.02260] [INSPIRE].

[20] K.B. Alkalaev and V.A. Belavin, Classical conformal blocks via AdS/CFT correspondence, JHEP 08 (2015) 049 [arXiv: 1504.05943] [INSPIRE].

[21] K.B. Alkalaev and V.A. Belavin, Monodromic vs geodesic computation of Virasoro classical conformal blocks, Nucl. Phys. B 904 (2016) 367 [arXiv:1510.06685] [InSPIRE].

[22] E. Hijano, P. Kraus, E. Perlmutter and R. Snively, Semiclassical Virasoro blocks from $A d S_{3}$ gravity, JHEP 12 (2015) 077 [arXiv: 1508.04987] [INSPIRE]. 
[23] E. Hijano, P. Kraus, E. Perlmutter and R. Snively, Witten Diagrams Revisited: The AdS Geometry of Conformal Blocks, JHEP 01 (2016) 146 [arXiv:1508.00501] [INSPIRE].

[24] A. Bhatta, P. Raman and N.V. Suryanarayana, Holographic Conformal Partial Waves as Gravitational Open Wilson Networks, JHEP 06 (2016) 119 [arXiv: 1602.02962] [INSPIRE].

[25] K.B. Alkalaev, Many-point classical conformal blocks and geodesic networks on the hyperbolic plane, JHEP 12 (2016) 070 [arXiv:1610.06717] [INSPIRE].

[26] V.A. Belavin and R.V. Geiko, Geodesic description of Heavy-Light Virasoro blocks, JHEP 08 (2017) 125 [arXiv: 1705.10950] [INSPIRE].

[27] P. Kraus, A. Maloney, H. Maxfield, G.S. Ng and J.-q. Wu, Witten Diagrams for Torus Conformal Blocks, JHEP 09 (2017) 149 [arXiv:1706.00047] [INSPIRE].

[28] K.B. Alkalaev and V.A. Belavin, Holographic duals of large-c torus conformal blocks, JHEP 10 (2017) 140 [arXiv:1707.09311] [INSPIRE].

[29] M. Nishida and K. Tamaoka, Fermions in Geodesic Witten Diagrams, JHEP 07 (2018) 149 [arXiv: 1805.00217] [INSPIRE].

[30] A. Bhatta, P. Raman and N.V. Suryanarayana, Scalar Blocks as Gravitational Wilson Networks, arXiv:1806.05475 [INSPIRE].

[31] Y. Hikida and T. Uetoko, Superconformal blocks from Wilson lines with loop corrections, arXiv: 1806.05836 [INSPIRE].

[32] A.L. Fitzpatrick, J. Kaplan and M.T. Walters, Virasoro Conformal Blocks and Thermality from Classical Background Fields, JHEP 11 (2015) 200 [arXiv: 1501.05315] [INSPIRE].

[33] Y. Kusuki, New Properties of Large-c Conformal Blocks from Recursion Relation, JHEP 07 (2018) 010 [arXiv:1804.06171] [INSPIRE].

[34] Y. Kusuki, Large c Virasoro Blocks from Monodromy Method beyond Known Limits, arXiv: 1806.04352 [INSPIRE].

[35] A.L. Fitzpatrick, J. Kaplan, Z.U. Khandker, D. Li, D. Poland and D. Simmons-Duffin, Covariant Approaches to Superconformal Blocks, JHEP 08 (2014) 129 [arXiv:1402.1167] [INSPIRE].

[36] H. Chen, A.L. Fitzpatrick, J. Kaplan, D. Li and J. Wang, Degenerate Operators and the $1 / c$ Expansion: Lorentzian Resummations, High Order Computations and Super-Virasoro Blocks, JHEP 03 (2017) 167 [arXiv: 1606. 02659] [INSPIRE].

[37] O. Aharony, L.F. Alday, A. Bissi and E. Perlmutter, Loops in AdS from Conformal Field Theory, JHEP 07 (2017) 036 [arXiv: 1612.03891] [INSPIRE].

[38] E. Perlmutter, Virasoro conformal blocks in closed form, JHEP 08 (2015) 088 [arXiv: 1502.07742] [INSPIRE].

[39] Y. Nakayama, Liouville field theory: A Decade after the revolution, Int. J. Mod. Phys. A 19 (2004) 2771 [hep-th/0402009] [INSPIRE].

[40] H. Poghosyan, The light asymptotic limit of conformal blocks in $\mathcal{N}=1$ super Liouville field theory, JHEP 09 (2017) 062 [arXiv: 1706.07474] [INSPIRE].

[41] K.B. Alkalaev and V.A. Belavin, From global to heavy-light: 5-point conformal blocks, JHEP 03 (2016) 184 [arXiv: 1512.07627] [INSPIRE].

[42] M. Cho, S. Collier and X. Yin, Genus Two Modular Bootstrap, arXiv:1705.05865 [INSPIRE]. 
[43] L. Hollands, C.A. Keller and J. Song, Towards a $4 d / 2 d$ correspondence for Sicilian quivers, JHEP 10 (2011) 100 [arXiv:1107.0973] [InSPIRE].

[44] K.B. Alkalaev, R.V. Geiko and V.A. Rappoport, Various semiclassical limits of torus conformal blocks, JHEP 04 (2017) 070 [arXiv: 1612.05891] [INSPIRE].

[45] K. Alkalaev and V. Belavin, Large-c superconformal torus blocks, JHEP 08 (2018) 042 [arXiv: 1805.12585] [INSPIRE].

[46] N. Javerzat, R. Santachiara and O. Foda, Notes on the solutions of Zamolodchikov-type recursion relations in Virasoro minimal models, arXiv:1806.02790 [INSPIRE].

[47] M.R. Gaberdiel, C.A. Keller and R. Volpato, Genus Two Partition Functions of Chiral Conformal Field Theories, Commun. Num. Theor. Phys. 4 (2010) 295 [arXiv:1002.3371] [INSPIRE].

[48] A. Belin, C.A. Keller and I.G. Zadeh, Genus two partition functions and Rényi entropies of large c conformal field theories, J. Phys. A 50 (2017) 435401 [arXiv:1704.08250] [InSPIRE].

[49] C.A. Keller, G. Mathys and I.G. Zadeh, Bootstrapping Chiral CFTs at Genus Two, arXiv: 1705.05862 [INSPIRE]. 